\title{
Relação entre estado nutricional, adiposidade corporal, percepção de autoimagem corporal e risco para transtornos alimentares em atletas de modalidades coletivas do gênero feminino
}

CDD. 20.ed. 302.222

796.5

\author{
Ana Claudia Pelissari KRAVCHYCHYN" \\ Danilo Fernandes da SILVA* \\ Fabiana Andrade MACHADO"
}

*Universidade Estadual

de Maringá.

\begin{abstract}
Resumo
0 objetivo do presente estudo foi analisar se há associação entre a autoimagem corporal, risco para transtornos alimentares, adiposidade corporal e estado nutricional em atletas de modalidades coletivas do gênero feminino. Participaram 45 atletas das modalidades de basquetebol, voleibol, handebol e futsal. Foram aferidas massa corporal, estatura e dobras cutâneas para a determinação do Índice de Massa Corporal (IMC) e percentual de gordura corporal (\%G). Os questionários aplicados foram o Body Shape Questionnarie (BSO) e o Eating Atittudes Test (EAT-26). A análise de associação foi feita pelo teste Qui-quadrado $2 \times 2$ e Exato de Fisher $(\mathrm{p}<0,05)$. A média do IMC e $\% \mathrm{G}$ foram de $22,82 \pm 2,73 \mathrm{~kg} \cdot \mathrm{m}^{-2}$ e $23,42 \pm 5,10 \%$, respectivamente. 0 BSO se associou com o IMC $(p=0,001)$ e com o \%G $(p=0,008)$, já EAT-26 não se associou com as variáveis antropométricas. Conclui-se que atletas em sobrepeso e obesidade possuem maior tendência a distorção da autoimagem corporal, porém não tem propensão para desenvolver transtornos alimentares.
\end{abstract}

Palavras-Chave: Composição corporal; Feminino; Questionários; Esportes.

\section{Introdução}

O corpo magro vem sendo preconizado como ideal de beleza gerando uma supervalorização da imagem corporal, norteando a busca por padrōes estéticos que nem sempre são necessariamente saudáveis $^{1-2}$. Essa supervalorização pode gerar um quadro de distorção da forma como o indivíduo se vê, e pode também estar associada a mudanças nos hábitos alimentares causando padrões restritivos da ingesta de alimentos e nutrientes ${ }^{2-4}$. Diante disso, é importante detectar se há grupos que estão mais suscetíveis a esses distúrbios.

DAMASCENO et al. ${ }^{5}$ mostraram que a insatisfação com a autoimagem corporal diminui com a idade. No gênero feminino, a preconização por um corpo magro é frequente em mulheres mais jovens possivelmente por conta das imposições socioculturais e padrões estéticos, tornando-as um grupo de risco para distorção da autoimagem corporal e transtornos alimentares ${ }^{5-6}$. Atletas de rendimento estão inseridas nesse grupo de risco, sendo que as mais afetadas são as atletas pertencentes a modalidades esportivas que preconizam o baixo peso corporal e altos padrōes estéticos como o balé e ginástica artística ${ }^{7-11}$.

RibeIro e VeIGA ${ }^{11}$ avaliaram transtornos alimentares e distorção da autoimagem corporal em 39 bailarinas e constataram que 10,3\% apresentaram risco para o desenvolvimento de transtornos alimentares. Somado a isso $66,7 \%$ das bailarinas apresentaram insatisfação com sua imagem corporal. VIEIRA et al. ${ }^{12}$ avaliaram 48 atletas de ginástica rítmica das categorias infantil e juvenil e encontraram correlação positiva entre a distorção da autoimagem corporal e a presença de transtornos alimentares para os dois grupos, sendo que $27,2 \%$ das atletas da categoria infantil e $20,0 \%$ das atletas da categoria juvenil apresentaram atitudes sugestivas de transtornos do comportamento alimentar. No entanto, pouca atenção tem sido dada a atletas de modalidades com caráter coletivo como o voleibol, basquetebol, handebol e futsal. 
Oliveira et al. ${ }^{9}$ e VIEIRA et al. ${ }^{13}$ apontaram que outras modalidades esportivas, como as modalidades coletivas, necessitam ter aspectos psicológicos avaliados (ex.: autoimagem corporal e transtornos alimentares), pois a causa multifatorial relacionada a esses distúrbios pode estar associada ao estilo de vida esportivo ou a pressão advinda dos padrões de corpo impostos pela sociedade, e não necessariamente a um esporte em específico, que tende a valorizar o corpo magro (ex.: ginástica rítmica, ginástica

\section{Método}

\section{Amostra}

Participaram do estudo 45 atletas do gênero feminino que competem em nível estadual, pertencentes a times da cidade de Maringá-PR, com idades entre 15 e 26 anos, praticantes das modalidades: voleibol (11 atletas), basquetebol (14 atletas), futsal (10 atletas) e handebol (10 atletas). Antes da avaliação as participantes foram esclarecidas sobre os procedimentos e assinaram o Termo de Consentimento Livre e Esclarecido. As atletas menores de idade, além de consentirem com a participação, também tiveram seus responsáveis consultados antes da realização do estudo. O projeto de pesquisa foi previamente aprovado pelo Comitê de Ética local (Parecer 687/2010).

\section{Procedimentos}

Foram aferidas medidas antropométricas referentes à massa corporal e estatura para determinação do Índice de Massa Corporal (IMC - $\mathrm{kg} \cdot \mathrm{m}^{-2}$ ) utilizado para classificação do estado nutricional das atletas adultas, segundo a World Health Organization ${ }^{14}$ e das atletas adolescentes, segundo os pontos de corte estabelecidos por ColE et al. ${ }^{15}$, de acordo com a idade e gênero da adolescente.

Também foram aferidas dobras cutâneas das regiōes biciptal, triciptal, peitoral, subescapular, supra-ilíaca, abdominal, coxa medial e panturrilha utilizando-se um compasso científico (Cescorf) para determinação do percentual de gordura $(\% \mathrm{G})$ a partir da fórmula de JACKSON e POLLOCK ${ }^{16}$. A amostra foi dividida "a posteriori" em dois grupos segundo o $\% \mathrm{G}$ para as análises de associação, sendo o percentil 50 (22,89\% de gordura corporal) utilizado como critério, tendo em vista que não há uma classificação pré-estabelecida para a população para esta variável. Assim, foi determinado artística, patinação, balé, etc.). Além disso, até onde temos conhecimento, não há estudos associando autoimagem corporal, transtornos alimentares e características antropométricas em mulheres praticantes de esportes coletivos.

O objetivo do presente estudo foi analisar se há relação entre a autoimagem corporal, transtornos alimentares, adiposidade corporal e estado nutricional de praticantes de modalidades coletivas do gênero feminino.

o grupo de atletas com os menores e com os maiores valores de \%G (Menores valores: 19,6 $\pm 2,8 \%$; Maiores valores: $27,5 \pm 3,6 \% ; \mathrm{p}<0,001$ ).

Foi aplicado o questionário Body Shape Questionnaire (Questionário de Imagem Corporal) - BSQ, traduzido por CORDÁs e NEVES ${ }^{17}$ e foi validado para a população brasileira por Di PIETRO ${ }^{18}$. Esse instrumento foi previamente utilizado para a população de atletas (ginástica rítmica), tanto para adolescentes como para adultos ${ }^{12}$ e busca identificar a preocupação do sujeito com a imagem corporal, identificando graus de distorção da autoimagem corporal, classificados como "ausência de distorção", "distorção leve", "distorção moderada" e "distorção grave". Para as análises inferenciais os dados foram agrupados em apenas duas categorias: "ausência de distorção" e "presença de distorção" (somatório de presença de distorção leve + moderada + grave).

Além disso, foi aplicado o Eating Atittudes Test (Teste de Atitudes Alimentares) - EAT-26, elaborado por GARNER et al. ${ }^{19}$, validado para a língua portuguesa por NunEs et al. ${ }^{20}$. Esse instrumento é utilizado para a detecção de indivíduos susceptíveis ao desenvolvimento de transtornos alimentares, sendo previamente aplicado em adolescentes ${ }^{20}$, adul$\operatorname{tos}^{21}$ e praticantes de modalidades esportivas, tanto adolescentes quanto adultos ${ }^{12}$. Com ele, os sujeitos foram classificados em "ausência de transtornos" e "presença de transtornos".

\section{Análise dos dados}

Inicialmente, foi aplicado o teste de Shapiro-Wilk para verificação da normalidade da distribuição dos dados. Para a análise descritiva dos dados, utilizou-se a média \pm desvio padrão (DP) para dados intervalares e as medidas de frequência absoluta e relativa para 
dados categóricos. A análise inferencial dos dados categóricos foi realizada a partir dos testes de associação Qui-quadrado $2 \times 2$. Caso a frequência esperada fosse menor que cinco em $20 \%$ ou mais das células, optouse pelo uso do Teste exato de Fisher. Foi adotado para todos os testes nível de significância de $\mathrm{p}<0,05$.

\section{Resultados}

$\mathrm{Na}$ TABELA 1 encontram-se os valores médios \pm desvio padrão (DP) das características gerais da amostra (Idade, anos; Massa Corporal, kg; Estatura, m; IMC, $\mathrm{kg} / \mathrm{m}^{2}$; Percentual de Gordura, \%G) de acordo com a modalidade esportiva.

Por não haver associação entre a modalidade esportiva praticada e presença de transtornos alimentares ( $p=0,201)$ e entre a modalidade e a distorção da autoimagem corporal $(\mathrm{p}=0,103)$, optou-se por realizar todas as demais análises de associação entre distorção da autoimagem corporal, presença de transtornos alimentares e os índices antropométricos com todas as atletas.

Além disso, não observamos associação entre a faixa etária (adolescente $(44,4 \%)$ ou adulta $(55,6 \%)$ ) com a autoimagem corporal $(\mathrm{p}=0,729)$ e com a presença de transtornos alimentares $(p=0,678)$. Desse modo, fizemos as análises com as todas as atletas em conjunto.

A TABELA 2 mostra não haver associação entre transtornos alimentares e distorção da autoimagem corporal. Com base nos dados dessa tabela, é possível observar que 11 atletas avaliadas (24,5\%) apresentaram algum grau de distorção da autoimagem corporal. Dessas, sete $(15,5 \%)$ apresentaram distorção leve e quatro (8,9\%), distorção moderada. Em relação à avaliação de transtornos alimentares, seis atletas $(13,3 \%)$ foram diagnosticadas com risco para o desenvolvimento de transtornos alimentares

TABELA 1 - Características gerais das atletas de acordo com a modalidade esportiva e no total.

\begin{tabular}{lccccc}
\hline & Total $(\mathbf{n}=\mathbf{4 5})$ & Voleibol $(\mathbf{n}=\mathbf{1 1})$ & Basquetebol $(\mathbf{n}=\mathbf{1 4})$ & Futsal $(\mathbf{n}=\mathbf{1 0})$ & Handebol $(\mathbf{n}=\mathbf{1 0})$ \\
Variável & Média \pm DP & Média \pm DP & Média \pm DP & Média \pm DP & Média \pm DP \\
\hline Idade & $18,84 \pm 3,07$ & $16,45 \pm 1,21$ & $19,00 \pm 3,16$ & $19,70 \pm 3,4$ & $20,40 \pm 2,8$ \\
Massa corporal $(\mathrm{kg})$ & $65,20 \pm 9,72$ & $68,84 \pm 10,11$ & $64,97 \pm 11,08$ & $60,33 \pm 5,96$ & $66,49 \pm 9,55$ \\
Estatura $(\mathrm{m})$ & $1,69 \pm 0,07$ & $1,74 \pm 0,05$ & $1,71 \pm 0,76$ & $1,64 \pm 0,04$ & $1,65 \pm 0,06$ \\
IMC $\left(\mathrm{kgm}^{-2}\right)$ & $22,82 \pm 2,73$ & $22,64 \pm 2,81$ & $22,03 \pm 2,49$ & $22,61 \pm 2,55$ & $24,35 \pm 2,94$ \\
$\% \mathrm{G}$ & $23,42 \pm 5,10$ & $25,44 \pm 3,92$ & $22,88 \pm 6,41$ & $20,83 \pm 4,38$ & $24,56 \pm 4,15$ \\
\hline
\end{tabular}

TABELA 2 - Tabela cruzada com a informação do risco para transtornos alimentares e distorção da imagem corporal em atletas do gênero feminino de modalidades coletivas.

\begin{tabular}{lccc}
\hline & \multicolumn{2}{c}{ Risco para transtornos alimentares } & P \\
& Ausente & Presente & \\
\hline Distorçáo da Imagem corporal & 31 & 3 & 0,146 \\
Ausência de distorção & 8 & 3 & \\
Presença de distorçáo & 39 & 6 & \\
Total & & & \\
\hline Teste exato de Fisher & & & \\
\hline
\end{tabular}

Como nenhuma atleta foi classificada com baixo peso ou obesidade, a análise inferencial foi feita com base em dois grupos: "peso normal" e "sobrepeso", sendo que da amostra avaliada, 10 (22,2\%) atletas estavam sobrepesadas. A distorção da autoimagem corporal associou-se com o estado nutricional e o percentual de gordura, conforme descrito na TABELA 3.

Com base nos resultados da TABELA 4 podese argumentar que a presença de transtornos alimentares não se associou com nenhuma das variáveis antropométricas. 
Os resultados do questionário BSQ apresentaram associação com as variáveis IMC e $\% \mathrm{G}$, em que $70 \%$ das atletas que foram classificadas com sobrepeso manifestaram algum tipo de distorção da autoimagem, assim como 50\% das atletas classificadas com o \%G acima de 22,89\%. Essa análise sugere que a distorção da autoimagem corporal é mais frequente em atletas com maior IMC e maior \%G.

TABELA 3 - Tabela cruzada com a informação do estado nutricional, adiposidade corporal e distorção da imagem corporal em atletas do gênero feminino de modalidades coletivas.

*Teste exato de Fisher. **Teste Qui-quadrado $2 \times 2$.

Teste exato de Fisher

\begin{tabular}{lccc}
\hline & \multicolumn{2}{c}{ Distorçáo da Imagem Corporal } & p \\
& Ausente & Presente & \\
\hline Estado Nutricional & & 4 & 0,001 \\
Peso Normal & 31 & 7 & \\
Sobrepeso & 3 & 11 & \\
Total & 34 & 2 & 0,012 \\
\hline Percentual de gordura** & & 9 & \\
Abaixo ou igual a 22,89\% & 21 & 11 & \\
Acima de $22,89 \%$ & 13 & & \\
Total & 34 & & \\
\hline
\end{tabular}

TABELA 4 - Tabela cruzada com a informação do estado nutricional, adiposidade corporal e risco para transtornos alimentares em atletas do gênero feminino de modalidades coletivas.

\begin{tabular}{lccc}
\hline & \multicolumn{2}{c}{ Risco para transtornos alimentares } & P \\
& Ausente & Presente & \\
\hline Estado Nutricional & & & \\
Peso Normal & 32 & 3 & 0,113 \\
Sobrepeso & 7 & 3 & \\
Total & 39 & 6 & \\
\hline Percentual de gordura** & & & \\
Abaixo ou igual a 22,89\% & 21 & 2 & \\
Acima de 22,89\% & 18 & 4 & \\
Total & 39 & 6 & \\
\hline
\end{tabular}

\section{Discussão}

O objetivo do presente estudo foi analisar se há relação entre a autoimagem corporal, transtornos alimentares, adiposidade corporal e estado nutricional de praticantes de modalidades coletivas do gênero feminino. $\mathrm{O}$ resultado das avaliações mostrou que $24,4 \%$ das atletas apresentaram distorção da autoimagem corporal havendo associação entre sobrepeso e a presença de distorção. Porém apenas seis $(13,3 \%)$ jogadoras apresentaram propensão para desenvolver transtornos alimentares, sendo que esta condição independe do estado nutricional e do \%G.

PeRINI et al. ${ }^{10}$ avaliaram a presença de transtornos alimentares em atletas de nada sincronizado das categorias júnior e sênior e verificaram que $11,1 \%$ das avaliadas apresentaram esse fator de risco. VIEIRA et al..$^{13}$ verificaram que $43,9 \%$ das adolescentes e adultas, praticantes de judô em nível estadual, apresentaram risco para o desenvolvimento de transtornos alimentares. Já VIEIRA et al. ${ }^{12}$ observaram prevalência de risco para transtornos alimentares em 16,6\% da amostra de atletas do gênero feminino de ginástica rítmica, também em adolescentes e adultas. A avaliação dos padrões alimentares revelou que $10,3 \%$ das mulheres praticantes de balé clássico apresentam conduta alimentar alterada ${ }^{11}$.

Em relação ao percentual de distorção da autoimagem corporal em atletas, $48,1 \%$ das praticantes 
de nado sincronizado apresentaram algum grau de distorção desse parâmetro ${ }^{10}$. Por outro lado $56,1 \%$ das atletas de judô possuem algum grau de distorção da autoimagem corporal ${ }^{13} \mathrm{e} 27,1 \%$ das atletas de ginástica rítmica algum grau de alteração para essa variável ${ }^{12}$.

Com exceção do estudo realizado com atletas de judô, em que a prevalência de risco para transtornos alimentares foi mais alta, os valores para risco de desenvolvimento de transtornos alimentares das praticantes de ginástica rítmica e nado sincronizado foram semelhantes aos verificados em atletas de modalidades coletivas, conforme observado no presente estudo. Para a distorção da autoimagem corporal, os valores de atletas de esportes coletivos foram mais baixos do que em praticantes de judô e nado sincronizado ${ }^{10,13}$, porém, foram próximos dos observados em atletas de ginástica rítmica ${ }^{12}$.

Os resultados encontrados são relevantes para o esporte de rendimento, pois é indesejável que um atleta que busca resultados positivos e superação de adversários, se envolva com problemas de bem estar emocional, desenvolvendo uma insatisfação com sua autoimagem corporal e, posteriormente, com o quadro agravado de distúrbios de comportamentos alimentares. De acordo com as análises, esses resultados independem da modalidade coletiva praticada (ex.: voleibol, basquetebol, futsal ou handebol), visto que não houve associação entre a modalidade com a presença de riscos para transtornos alimentares e distorção da autoimagem corporal. Esperava-se esse resultado, pois o cenário/ambiente dos esportes coletivos é semelhante entre as modalidades, o que poderia levar a respostas semelhantes em relação a essas variáveis psicológicas. Outra possibilidade de interpretação é que independente da modalidade praticada, todas são mulheres jovens, situação em que a preconização por um corpo magro é frequente por conta das imposiçôes socioculturais e padrões estéticos, tornando-as um grupo de risco para distorção da autoimagem corporal e transtornos alimentares ${ }^{5-6}$.

Apesar do percentual de atletas com risco para transtornos alimentares e distorção da autoimagem corporal em praticantes de modalidades coletivas serem próximos ao observado em outros grupos de atletas considerados de maior risco ${ }^{22}$, não observamos associação entre essas duas variáveis no presente estudo. Em atletas de ginástica rítmica, VIEIRA et al. ${ }^{12}$ também concluíram que não houve associação entre essas duas variáveis. Entretanto, há estudos que apontam a distorção da autoimagem corporal como um fator de risco para o desenvolvimento de transtornos de conduta alimentar ${ }^{23-24}$. Alves et al..$^{25}$ verificaram que adolescentes insatisfeitos com sua imagem corporal tem 16,7 vezes mais chance de desenvolver transtornos alimentares do que aqueles que estão satisfeitos. VIEIRA et al. ${ }^{13}$ demonstraram haver correlação entre essas duas variáveis.

Portanto, mesmo que seja de grau leve, a distorção da autoimagem corporal deve ser levada em consideração em diferentes contextos, inclusive no das modalidades coletivas, haja vista que a pressão social diária por um corpo magro é observada, de modo geral, em adolescentes e adultas jovens, mesmo que não sejam atletas ${ }^{9,12,26-27}$.

Fatores como a percepção comportamental e cognitiva individual em relação às exigências do ambiente, podem melhor explicar a presença de distorção da autoimagem corporal e de transtornos alimentares ${ }^{28}$. Além desses, é possível destacar o excesso de peso ou excesso de gordura corporal, pois se o sujeito com peso normal pode já apresentar descontentamento com seu corpo ${ }^{29}$, a pressão social é ainda maior sobre aqueles que estão acima do peso.

Considerando a característica do gênero e da fase em que estão passando, estudos verificaram que adolescentes do gênero feminino tendem a superestimar seu estado nutricional ${ }^{2,26}$, apesar de ELGAR et al. ${ }^{30}$ terem verificado uma subestimação do real estado nutricional em ambos os gêneros. FrIEDMAN e BROWNELL ${ }^{31}$ demonstraram, em sua revisão, que há associação entre excesso de peso e distorção da autoimagem corporal; entretanto, essa relação é menos consistente do que a relação entre insatisfação com a imagem corporal e excesso de peso.

A presença mais frequente de distorção da autoimagem corporal nas atletas com excesso de peso pode estar associada a uma manifestação de insegurança, ansiedade, assim como uma maior dificuldade em se adaptar ao ambiente ${ }^{32}$. Os resultados deste estudo sugerem que, mesmo sendo praticantes de modalidades esportivas em nível competitivo, capazes de realizar tarefas motoras mais complexas, as atletas apresentam alterações psicossociais. Ou seja, conforme já discutido, os reflexos dos padrões sociais pré-estabelecidos parecem ser mais fortes, e aspectos como a prática de modalidades esportivas, não seriam fatores de proteção contra a distorção da autoimagem corporal.

Apesar disso, a presença de transtornos alimentares não se associou com o estado nutricional ou a adiposidade corporal. Até onde temos conhecimento, não há descrito na literatura essa relação em atletas. No entanto, em universitários, não houve associação entre ser sobrepesado e apresentar maior risco para transtornos alimentares, semelhante ao observado 
para as praticantes de modalidades coletivas. Apenas o estrato de obesos, a partir da classificação do IMC, apresentou relação com comportamentos bulímicos ${ }^{33}$.

Diante do exposto, tantos os distúrbios de autoimagem como de alimentação apresentam importantes fatores a serem observados no grupo abordado, pois desencadeiam consequências graves que, se não diagnosticados e tratados, podem trazer problemas sérios à vida pessoal e ao bom rendimento do trabalho diário de atletas que desenvolvem essas patologias $^{12}$.

Uma limitação do presente estudo foi o número reduzido de atletas de modalidades coletivas, apesar de terem sido avaliadas todas as equipes que competem em nível estadual na cidade de Maringá-PR. Também não foi verificada a influência do tempo de prática da modalidade sobre as variáveis mensuradas. Além disso, a autoimagem corporal foi classificada de maneira dicotômica, podendo haver diferenças em relação ao grau de distorção (ex.: leve, moderado e grave).
Com base no presente estudo pôde-se notar que em atletas do gênero feminino, praticantes de modalidades coletivas, houve associação entre a distorção da autoimagem corporal, excesso de peso e maior adiposidade corporal. Entretanto, a distorção da autoimagem corporal não se associou com a presença de transtornos alimentares, excesso de peso e maior adiposidade corporal. Portanto, conclui-se que atletas de modalidade coletiva em sobrepeso e obesidade possuem maior tendência a distorção da autoimagem corporal, porém não tem propensão para desenvolver transtornos alimentares. Sugere-se a realização de estudos com atletas de nível nacional e internacional que tem uma rotina muito mais vinculada ao esporte e que estão frequentemente aparecendo em diferentes veículos de comunicação que costumam expor seu corpo perante a população para verificar o impacto dessa prática sobre a autoimagem corporal e a presença de transtornos alimentares.

\begin{abstract}
Association between nutritional status, body adiposity, body self-image and risk for eating disorders in female college students athletes of collective sports

The objective of the present study was to analyze wheter there is association between body self-image, risk for eating disorders, body fat, and BMI classification in collective sports female athletes. Forty-five basketball, volleyball, handball and futsal athletes participated in the research. We measured weight, height and skinfolds were measures for determining the Body Mass Index (BMI) and Body Fat Percentage (\%fat). It was used the questionnaires Body Shape Questionnaire (BSO) and Eating Attitudes Test (EAT-26). The association analysis was done by Chi-square $2 \times 2$ and Fisher's Exact Test $(p<0.05)$. The mean BMI and \%fat were $22.82 \pm 2.73$ $\mathrm{kg} \cdot \mathrm{m}^{-2}$ and $23.42 \pm 5.10 \%$, respectively. The BSO was associated with BMI $(p=0.001)$ and \%fat $(p=0.008)$ and EAT-26 was not associated with any anthropometric variables. It was concluded that overweight and obese athletes had a greater tendency to body self-image distortion, but these athletes did not have the propensity to develop eating disorders.
\end{abstract}

KEY WORDS: Body composition; Female; Questionnaires; Sports.

\title{
Referências
}

1. Morgado FFR, Ferreira MEC, Andrade MRM, Segheto KJ. Analysis of the body image evaluation instruments. Fit Perf J. 2009;8:204-11.

2. Araujo CL, Dumith SC, Menezes AMB, Hallal PC. Peso medido, peso percebido e fatores associados em adolescentes. Rev Panam Salud Publica. 2010;27:360-7.

3. Russo R. Imagem corporal: construção através da cultura do belo. Mov percepc. 2005;5:80-90.

4. De Cicco MF, Santos N, Silva MM, Laham C, Garrido Junior A, Lucia MC. Imagem corporal, práticas de dietas e crenças alimentares em adolescentes e adultas. Psicol Hosp. 2006;4:1-27. 
5. Damasceno VO, Vianna VRA, Vianna JM, Lacio M, Lima JRP, Novaes JS. Imagem corporal e corpo ideal. Rev Bras Cienc Mov. 2006;14:87-96.

6. Damasceno VO, Lima JRP, Vianna JM, Vianna VRA, Novaes JS. Tipo físico ideal e satisfação com a imagem corporal de praticantes de caminhada. Rev Bras Med Esporte. 2005;11:181-6.

7. Zucker P. Eating disorders in young athletes: a round table. Phys Sportsmed. 1985;13, p. 89-106, 1985.

8. Wilmore JH. Eating and weight disorders in the female athlete. J Int Soc Sports Nutr. 1991;1:104-15.

9. Oliveira FP, Bosi MLM, Vigário PS, Vieira RS. Comportamento alimentar e imagem corporal em atletas. Rev Bras Med Esporte. 2003;9:348-56.

10. Perini T, Vieira R, Vigario P, Oliveira G, Ornellas J, Oliveira F. Transtornos do comportamento alimentar em atletas de elite do nado sincronizado. Rev Bras Med Esporte. 2009;15:54-7.

11. Ribeiro LG, Veiga GV. Imagem corporal e comportamentos de risco para transtornos alimentares em bailarinos profissionais. Rev Bras Med Esporte. 2010;16:99-102.

12. Vieira JLL, Amorim HZ, Vieira LF, Amorim AC, Rocha PGM. Distúrbios de atitude alimentares e distorção da imagem corporal no contexto competitivo da ginástica rítmica. Rev Bras Med Esporte. 2009;15:410-4.

13. Vieira JLL, Oliveira LP, Vieira LF, Vissoci JRN, Hoshino EF, Fernandes SL. Distúrbios de atitudes alimentares e sua relação com a distorção da auto-imagem corporal em atletas de judô do Estado do Paraná. Rev Educ Fís/UEM. 2006;17:177-84.

14. WHO. World Health Organization. Report of a WHO consultation on obesity. Obesity: preventing and managing the global epidemic. Geneva: World Health Organization; 1998.

15. Cole TJ, Bellizzi MC, Flegal KM, Dietz WH. Establishing a standard definition for child overweight and obesity worldwide: international survey. Br Med J. 2000;320:1240-3.

16. Jackson A, Pollock M, Ward A. Generalized equations for predicting body density of women. Med Sci Sports Exerc. 1980;12:175-82.

17. Córdas TA, Neves JE. Escalas de avaliação dos transtornos alimentares. Rev Psiquiatr Clin. 1999;26:41-7.

18. Di Pietro MC. Validade interna, dimensionalidade e desempenho da escala BSQ - "Body Shape Questionnaire” em uma população de estudantes universitários [dissertação]. São Paulo (SP): Universidade Federal de São Paulo, Escola Paulista de Medicina; 2002.

19. Garner DM, Olmsted MP, Polivy J. Development and validation of a multidimensional eating disorder inventory for anorexia nervosa and bulimia. Int J Eat Disord. 1982;2:14-34.

20. Nunes MAA, Bagatini LF, Abuchaim ALG. O teste de atitudes alimentares (EAT-26) em adolescentes de Porto Alegre. Arq Psiquiatr Psicoter Psicanal. 1994;1:132-7.

21. Alvarenga MS, Scagliusi FB, Philippi ST. Comportamento de risco para transtorno alimentar em universitárias brasileiras. Rev Psiquiatr Clin. 2011;38:3-7.

22. Sundgot-Borgen J, Torstveit M. Prevalence of disorders in elite athletes in higher than in the general population. Clin J Sport Med. 2004;14:25-32.

23. Haase AM, Prapavessis H, Owens RG. Perfectionism, social physique anxiety and disordered eating: a comparison of male and female elite athletes. Psychol Sport Exerc. 2002;3:209-22.

24. Patel DR, Grydanus DE, Pratt HD, Phillips EL. Eating disorders in adolescents athletes. J Adolesc Res. 2003;18:280-9.

25. Alves E, Vasconcelos FAG, Calvo MCM, Neves J. Prevalência de sintomas de anorexia nervosa e insatisfação com a imagem corporal em adolescentes do sexo feminino do município de Florianópolis, Santa Catarina, Brasil. Cad Saude Publica. 2008;24:503-12.

26. Branco LM, Hilário MOE, Cintra IP. Percepção e satisfação corporal em adolescentes e a relação com seu estado nutricional. Rev Psiquiatr Clin. 2006;33:292-6.

27. Nicolaou M, Doak C, Dam R, Hosper K, Seidell J, Stronks K. Body size preference and body weight perception among two migrant groups of non-Western origin. Public Health Nutr. 2008;11:1332-41.

28. Dale KS, Landers DM. Weight control in wrestling: eating disorders or disordered eating? Med Sci Sports Exerc. 1999;31:1382-9.

29. Pereira ÉF, Graup S, Lopes AS, Borgatto AF, Daronco LSE. Percepção da imagem corporal de crianças e adolescentes com diferentes níveis socioeconômicos na cidade de Florianópolis, Santa Catarina, Brasil. Rev Bras Saude Mater Infant. 2009;9:253-62.

30. Elgar FJ, Roberts C, Tudor-Smith C, Moore L. Validity of self-reported height and weight and predictors of bias in adolescents. J Adolesc Health. 2005;37:371-75. 
Kravchychyn AC, et al.

31. Friedman MA, Brownell KD. Psychological correlats of obesity: moving to the next research generation. Psychol Bull. 1995; 117:3-20.

32. Almeida GAN, Loureiro SR, Santos JE. A imagem corporal de mulheres morbidamente obesas avaliada através do desenho da figura humana. Psicol Reflex Crit. 2002;15:283-92.

33. Cenci M, Peres KG, Vasconcelos FAG. Prevalence of bulimic behavior and associated factors in undergraduate female students. Rev Psiquiatr Clin. 2009;36:83-8.

\section{Agradecimentos}

Fundação Araucária (Processo 4287/2010).

ENDEREÇO

Fabiana Andrade Machado Universidade Estadual de Maringá Av. Colombo, 5790 - Jd. Universitário 87020-900 - Maringá - PR - BRASIL e-mail: famachado_uem@hotmail.com
Recebido para publicação: 27/04/2012

1a. Revisão: 28/03/2013

2a. Revisão: 10/04/2013

Aceito: $18 / 04 / 2013$ 